\title{
Postacquisition unexpected footshock disrupts appetitively motivated instrumental performance based on short-term retention
}

\author{
RALPH R. MILLER and MARY ANN BALAZ \\ State University of New York, Binghamton, New York
}

\begin{abstract}
Water-deprived rats were trained to run for water in an E-maze on a delayed-alternation task with a 5-min retention interval. Each day, long before encountering and again long after having encountered the delayed-alternation task, all subjects consistently experienced weak footshock in Context A and no footshock in Context B. Cu-texts A and B were highly dissimilar to each other and to the E-maze. On delayed-alternation probe trials, subjects were exposed to Context A or Context B for the middle $30 \mathrm{sec}$ of the delayed-alternation retention interval. Performance on these trials was unaffected when weak footshock occurred in Context A or no footshock occurred in Context B. However, when weak footshock occurred in Context B, retention was impaired. An absence of footshocks in Context A produced a nonsignificant tendency towards impairment. Thus, the disruptive effects of unexpected events that occur during the retention interval do not appear to depend upon the target and unexpected events' having similar reinforcers or even having reinforcers of the same valence. The results are interpreted in terms of unexpected events' recruiting more of the limited information-processing capacity of the subject than do otherwise similar expected events. Moreover, the tendency of unexpected shock to interfere with performance more than does unexpected nonshock suggests that the degree to which an unexpected event recruits processing capacity depends on the affective value of the event as well as on its unexpectedness.
\end{abstract}

In a recent study of delayed alternation with rats, our laboratory found that an intervening event during the retention period disrupted test performance through two independent, but additive, types of retroactive interference (Miller, Greco, Marlin, \& Balaz, Note 1). The first, similarity interference, depended, independently of the interval between the two events, upon the similarity of the target event and the intervening event and presumably arose from some sort of confusion between the two events when the memory trace of the intervening event was being established and/or when retrieval of the target trace was attempted. The second type of interference, processing interference, depended, independently of the similarity of the two traces, upon the interval between the intervening event and the target event and presumably arose from some sort of competition between the two traces for the limited processing capacity of the organism. Implicit in this view of processing interference is that target retention will decrease as the processing demands of the intervening event increase.

It is often assumed that an event will be processed to the degree that it is ecologically relevant and to the

This research was supported in part by NIMH Grant 33881 . We are grateful to Wesley J. Kasprow and Todd R. Schachtman for their critical reading of an earlier version of the manuscript. Reprint requests should be directed to Ralph R. Miller, Department of Psychology, SUNY-Binghamton, Binghamton, New York 13901. extent that it provides new information, that is, is unexpected or "surprising." Blanchard and Honig (1976), Grant, Brewster, and Stierhoff (1983), Kamin (1969), Maki (1979), and Terry and Wagner (1975) have all reported that surprising events are better remembered than expected events, presumably reflecting more extensive processing. Moreover, numerous students of human cognition (e.g., Brown, 1958; Peterson \& Peterson, 1959) have argued that one event will interfere with the processing of another to the degree that the former strains the (presumably) limited processing capacity of the organism. Integrating the notion that surprise enhances processing with the view that retention can be impaired by a nontarget event that overloads a limited processing capacity, Kremer (1979) and Wagner, Rudy, and Whitlow (1973) demonstrated that an unexpected, salient intervening nontarget US presentation interfered with manifest retention of a temporally proximal target CS-US pairing more than did an otherwise comparable expected intervening event. In both of these studies, footshock served as the US in the intervening event as well as in the target event.

The present research was designed to test further the finding that processing interference with the target trace is enhanced when the intervening event is unexpected, and in particular to examine the generality of this finding by using target and intervening reinforcers of opposite valence. Specifically, we employed an instrumental target task rather than a classical conditioning 
procedure such as that used by Kremer (1979) and Wagner et al. (1973), and we selected a target-event reinforcer (water) that was highly dissimilar and opposite in valence to the intervening-event reinforcer (footshock). This choice of an intervening event contrasted with our previous investigation of processing interference (Miller et al., Note 1), in which the intervening event consisted of novel information (unrelated to the appetitive target event) lacking any clearly appetitive or aversive characteristics. In this prior research, the salience of the intervening unexpected event was established by the intensity of the constituent audiovisual stimuli rather than by any strong affective consequences. In the present research, the footshock level was kept relatively low $(.5 \mathrm{~mA})$ because prior (unpublished) research in our laboratory had used the same preparation and had found that stronger footshock $(.75 \mathrm{~mA})$ administered outside the test apparatus between training and testing was disruptive to short-term retention whether or not the shock was surprising.

\section{METHOD}

\section{Subjects}

Twelve naive male albino rats of the Sprague-Dawley strain (CD animals from Charles River Breeding Laboratories, Wilmington, Massachusetts) served as subjects. The animals weighed 200-390 $\mathrm{g}$ at the initiation of the study. All rats were individually housed in cages made of wire mesh and sheet metal. Purina Laboratory Chow pellets were available ad lib, and water was accessible $10 \mathrm{~min} /$ day approximately $60 \mathrm{~min}$ after each day's experimental manipulation. The animals were acclimated gradually to this deprivation schedule and were handled daily during the week prior to the onset of the study.

\section{Apparatus}

The apparatuses were two identical E-mazes. One maze was used for acquisition trials and the other maze was used for test trials, thereby eliminating the possibility that animals would respond on test trials by using odor trails laid down during the preceding acquisition trial. In each maze, the start compartment was $27.5 \mathrm{~cm}$ long, the transverse alley was $62 \mathrm{~cm}$ long, and the arms containing the goalboxes were $19 \mathrm{~cm}$ long. All maze sections were $7 \mathrm{~cm}$ wide and $12.5 \mathrm{~cm}$ high, with stainless steel rods $.3 \mathrm{~cm}$ in diameter forming a grid floor. The walls, ceilings, and doors between compartments were made of clear Plexiglas. The walls of one goal compartment and the half of the transverse alley leading to it were covered externally with white paper, the walls of the other goal compartment and the other half of the transverse maze section were covered similarly with black paper, and the start compartment walls were transparent. In each maze at the far end of both goal compartments, there was a glass dish $(4.5 \mathrm{~cm}$ in diameter and $1.5 \mathrm{~cm}$ deep) filled with water; however, access to the water in one of the two goal compartments in each maze was blocked on test trials by a clear Plexiglas barrier containing a number of holes $(1.25 \mathrm{~cm}$ in diameter) that allowed the passage of any odor that the water may have emitted. Six lights served as discriminative stimuli. These lights, No. 1819 bulbs covered with translucent white lens caps and mounted $2 \mathrm{~cm}$ above the floor, were positioned $4 \mathrm{~cm}$ to the right and left of the center of the maze transverse wall facing the start compartment, at the center of the right and left ends of the maze transverse (i.e., at the entrances to the goalboxes), and at the center of the far end of both goalboxes immediately above the water dishes. On any given trial, either the right three or left three lights were on. The illumination conditions in the two mazes were independent of one another; that is, left- or right-side illumination of one maze was equally probable given left- or right-side illumination of the other maze. The experimental room was dimly illuminated and a $70-\mathrm{dB}(\mathrm{C})$ white noise served as an auditory background.

A holding cage similar in construction to the subjects' home cages was also in the experimental room in order to restrain the animals during the delay intervals. The holding cage contained no food or water.

Intervening treatments were given in two enclosures that were highly dissimilar to either the E-maze or the holding cage. One enclosure consisted of a conventional Grason-Stadler operant chamber, with the lever removed, that was housed in a dark environmental chest with a 61-dB(C) fan-generated noise level. The floor of this enclosure was composed of .2-cm-diam stainless steel rods that were serially connected by NE-2 neon bulbs. The other enclosure was a $52 \times 50 \mathrm{~cm}$ open field with walls of aluminum and brown Plexiglas and a floor composed of .4-cm-diam stainless steel rods that were serially connected by NE-2 neon bulbs. The open field was diffusely illuminated in the same way as the maze arms with the cue lights off, and it contained the same white-noise level as the maze. Thus, the open field differed from the operant chamber in terms of enclosure size, grid diameter, illumination, and auditory stimulation. On a random basis, half of the animals were assigned the operant chamber as the location for expected shock and the open field as the location for expected nonshock. For the other half of the animals, these assignments were reversed.

\section{Procedure}

The basic delayed-alternation task was modeled after that of Gordon and Feldman (1978) and Grant (1981).

Shaping was achieved in the following manner. On Day 1, each subject received 15 min of adaptation to each of the two mazes (all doors open), including unrestricted access to both of the water dishes in each maze. During this time, all maze doors remained open and all six cue lights were illuminated. Also, during this time, each animal visited all parts of the two mazes and drank from each water dish. Training began on Day 2 and consisted of 10 pairs of trials per day, with 10-30 min between pairs. Each pair of trials consisted of a forced-choice trial (target event) followed by a free-choice trial. Forced trials consisted of placing an animal in the start compartment of the acquisition maze facing away from the choice point; a clear Plexiglas door inserted perpendicularly to the transverse at the choice point forced the animal to a goalbox that was randomly chosen for each animal on each forced trial. On Day 2, only the three cue lights on the side of the maze to which the rat was forced were illuminated. Upon reaching the accessible water dish, the animal was allowed to drink for $10 \mathrm{sec}$. Then it was placed in the start compartment of the test maze and was allowed access to both goal compartments, but the perforated door prevented its drinking from the water dish in one randomly selected arm of the maze. Only the three cue lights on the side of the maze with the inaccessible goalbox were illuminated; that is, alternation of illumination condition was rewarded. A choice was recorded when an animal's head reached the entrance to a goalbox; incomplete trips in the transverse alley prior to the animal's making a choice were ignored. If the animal chose the reinforced arm, it was allowed to drink for $10 \mathrm{sec}$ and was then returned to its home cage. However, if the animal failed to alternate with respect to the forced trial, it was immediately given repeated correction trials until it reached the accessible water dish and drank for $10 \mathrm{sec}$; that is, it was removed from the incorrect arm and placed back in the startbox. After an interval of 10-30 min in the home cage had passed, another pair of forced and free trials was given; this continued until each animal had received a total of 10 pairs of trials. The procedure of Day 2 continued over successive days until a subject responded correctly on 8 of 10 consecutive free trials within a single day. Beginning on the day after this criterion had been reached, the animals were 
forced into the nonilluminated arm of the acquisition maze on each of the 10 daily forced trials. Now water on the free-choice trial was accessible on the illuminated side of the test maze. After again having achieved a criterion of 8 of 10 correct, each subject was switched to a condition in which the side of the acquisition maze on which the cue lights were illuminated, forced arm or blocked arm, was independently determined on each forced and again on each free trial rather than being constant within days. As before, on the free trials, alternation with respect to the illumination condition of the immediately preceding forced trial was reinforced. After a subject once again had reached the 8-of-10-correct criterion, the procedure was repeated, with a 15-sec delay (in the holding cage) between the forced and the free trial that constituted a trial pair. Each time a rat reached the daily 8 -of-10-correct criterion, the delay was increased, first to $30 \mathrm{sec}$ and then to $60 \mathrm{sec}$. When criterion had been reached with the 60 -sec delay, the number of trials per day was reduced to 5 and the delay was further increased for each subject, sequentially, to $1.5,2,3,4$, and $5 \mathrm{~min}$ on the day following the subject's having achieved a 4-of-5-correct criterion. When this criterion had been reached with a 5-min delay, the number of trial pairs per day for that animal was reduced to 1 and the use of correction trials was terminated.

Starting at this time, on each day before its forced trial, each animal was placed in its shock enclosure (operant chamber or open field) for $33 \mathrm{sec}$ and was given .5-mA constant-current footshock for the last $30 \mathrm{sec}$ of this period, after which it was returned to its home cage. Between 5 and $60 \mathrm{~min}$ later, it was placed in its nonshock enclosure (open field or operant chamber) for $33 \mathrm{sec}$, during which time it was given no shock. After another 5-60 $\mathrm{min}$ in its home cage, each animal was given its daily forced trial, followed immediately by a 5 -min retention inte: val in the holding cage and then the free-choice trial, before it was returned to its home cage. Between 5 and $60 \mathrm{~min}$ later, it was again given $30 \mathrm{sec}$ of shock in its designated shock enclosure, followed by 5-60 min in its home cage, and finally $30 \mathrm{sec}$ of no shock in its nonshock enclosure, before it was returned to its home cage for the remainder of the day.

Also beginning at this time, a probe-trial pair occurred on the day following an animal's completion of five successive correct responses (across days). By the first probe trial, all animals had received a minimum of 11 exposures to the shock enclosure paired with footshock and 11 exposures to the nonshock enclosures paired with the absence of footshock. To maintain expectancies with respect to Contexts $A$ and $B$, these pretrial and posttrial shock and nonshock exposures continued through to the conclusion of the experiment. A probe-trial pair differed from an ordinary-trial pair only in that the subject was placed either in the shock enclosure with or without shock or in the nonshock enclosure with or without shock for the middle $30 \mathrm{sec}$ of the retention interval. Thus, the four intervening treatments consisted of expected shock, expected nonshock, unexpected shock, and unexpected nonshock.

The experiment was effectively terminated 35 days after the animals had begun receiving exposure to the shock and nonshock enclosures. This limit was imposed because the frequency of errors decreased precipitously starting 35 days after completion of shaping; that is, there was a practice effect, and after 35 days the likelihood of further errors on probe trials was so low as to reduce the sensitivity of the probe trials. During the first 35 days of shock and nonshock experience, the error rate on probe trials remained constant at about $25 \%$; however, after the 35 th day, the error rate on probe trials fell rapidly to below $10 \%$. The average number of probe trials received by an animal during these 35 running days was 3.08 , with no animal serving more than once in any of the four probe conditions. Counterbalancing the order of exposure to different types of probe trials kept the practice effect from biasing the data. Choice behavior from postshaping days without retention-interval treatment was pooled to provide an index of baseline behavior.

\section{RESULTS}

The average rate of correct responding on nonprobe trials was $82 \%$. On shock probe trials, $92 \%$ of the responses were correct when the shock was expected and $57 \%$ were correct when the shock was unexpected. On nonshock probe trials, $88 \%$ of the responses were correct when the nonshock was expected and $70 \%$ were correct when the nonshock was unexpected. Because each subject did not serve in all conditions and because there was no correlation between conditions of individual subjects' performance on probe trials, statistics appropriate for independent samples were used to analyze the data. Ferguson's (1976) test for significance of the difference between independent proportions found that the unexpected probe trials produced more interference than the expected probe trials $[Z=2.20, p<.03$, two-tailed], whereas the shock probe trials proved no more disruptive than the nonshock trials $[\mathrm{Z}=.29, \mathrm{p}>.77]$. The difference between expected and unexpected probe trials appeared to derive primarily from unexpected shock's being more disruptive than expected shock $[\mathrm{Z}=2.18, \mathrm{p}<.03]$, rather than from unexpected nonshock's being more disruptive than expected nonshock $[\mathrm{Z}=.85, \mathrm{p}>.60]$. An analysis of performance on each type of probe trial as a function of when it occurred in the sequence of probe trials found no significant differences (all ps $>.2)$

\section{DISCUSSION}

The precipitous decline in interference seen after approximately 35 days (excluding 10 days of shaping) suggests that, with repetition, the alternating task became easier, perhaps "automatic" (Shiffrin \& Schneider, 1977), and, hence, less vulnerable to interference. Alternatively, it is possible that, rather than the target task's becoming effectively easier, the interfering events, through repetition, became less deleterious, that is, with repetition, lost their unexpectedness. In other words, the animals may have learned that, in a given enclosure, a particular shock condition prevailed most of the time but that the other shock condition did occasionally occur. However, this alternative loses credibility because of the lack of any sequential effects and because no animal was exposed to the same unexpected intervening event more than once.

Earlier unpublished research in our laboratory had found that stronger footshock during the retention interval than that used in the present study resulted in disruption of delayed-alternation performance whether or not the shock was unexpected. The current lack of any resultant difference between nonsurprising shock and nonsurprising nonshock treatments during retention intervals suggests, to the extent that cross-experiment comparisons can, that the weaker shock that was used in this study prevented expected shock from interfering with test performance, thereby making the study more sensitive to the disruptive effects of unexpected events during the retention interval than our earlier study had been.

Turning to the central issue of this experiment, the observed difference between expected and surprising intervening reinforcers is consistent with the view that unexpected events demand more processing than do expected events, thereby leaving less processing capacity for the target information (Kremer, 1979; Wagner et al., 1973). Moreover, the present data demon- 
strate that this effect does not depend on the target task and the surprising event having the same valence, an issue that was not addressed by Kremer or Wagner et al. That this difference stemmed largely from the disruptive effects of unexpected shock, as opposed to unexpected nonshock, suggests that the amount of processing depends not only upon the intervening event's being unexpected, but also upon the affective importance of the event, that is, its salience. The present failure of unexpected nonshock to disrupt retention appears to be inconsistent with the findings of Kremer and Wagner et al. that unexpected nonshock was as disruptive as unexpected shock. On the one hand, the basis of this discrepancy is not immediately clear. On the other hand, the current study did in fact produce a slight, albeit highly nonsignificant, tendency towards unexpected nonshock during the retention interval being more disruptive than expected nonshock. Perhaps with somewhat modified parameters this tendency could be enhanced. A likely alteration to achieve this effect would be an increase in shock intensity; however, such a modification, if too great, would probably eliminate the difference between expected and unexpected shock. Alternatively, the great qualitative dissimilarity between the present instrumental target task and the Pavlovian preparations of Kremer and Wagner et al. may have resulted in sensitivity to different aspects of the intervening event such that reinforcement versus nonreinforcement was critical to disruption by unexpected events in the present case, but not to disruption in the situations that these earlier investigators created.

\section{REFERENCE NOTE}

1. Miller, R. R., Greco, C., Marlin, N. A., \& Balaz, M. A. Retroactive interference in rats: Two independent processes. Manuscript submitted for publication, 1983.

\section{REFERENCES}

Blanchard, R., \& Honig, W. K. Surprise value of food determines its effectiveness as a reinforcer. Journal of Experimental Psychology: Animal Behavior Processes, 1976, 2, 67-74.

Brown, J. A. Some tests of the decay theory of immediate mem- ory. Quarterly Journal of Experimental Psychology, 1958, 10, 12-21.

Ferguson, G. A. Statistical analysis in psychology and education (4th ed.). New York: McGraw-Hill, 1976.

Gordon, W. C., \& Feldman, D. T. Reactivation-induced interference in a short-term retention paradigm. Learning and Motivation, 1978, 9, 164-178.

GRANT, D. S. Intertrial interference in rat short-term memory. Journal of Experimental Psychology: Animal Behavior Processes, 1981, 7, 217-227.

Grant, D. S., Brewster, R. G., \& Stierhoff, K. A. "Surprisingness" and short-term retention in pigeons. Journal of Experimental Psychology: Animal Behavior Processes, 1983, 9, 63-79.

Kamin, L. J. Predictability, surprise, attention, and conditioning. In B. A. Campbell \& R. M. Church (Eds.), Punishment and aversive behavior. New York: Appleton-Century-Crofts, 1969.

Kremer, E. F. Effect of posttrial episodes on conditioning in compound conditioned stimuli. Journal of Experimental Psychology: Animal Behavior Processes, 1979, 5, 130-141.

MAKI, W. S. Pigeons' short-term memories for surprising vs. expected reinforcement and nonreinforcement. Animal Learning \& Behavior, 1979, 7, 31-37.

Peterson, L., \& Peterson, M. J. Short-term retention of individual verbal items. Journal of Experimental Psychology, 1959, 58, 193-198.

Shiffrin, R. M., \& Schneider, W. Controlled and automatic human information processing: II. Perceptual learning, automatic attending, and a general theory. Psychological Review, 1977, 84, 129-190.

Terry, W. S., \& Wagner, A. R. Short-term memory for "surprising" versus "expected" unconditioned stimuli in Pavlovian conditioning. Journal of Experimental Psychology: Animal Behavior Processes, 1975, 1, 122-133.

Wagner, A. R., Rudy, J. W., \& Whitlow, J. W. Rehearsal in animal conditioning. Journal of Experimental Psychology, 1973, 97, 407-426.

(Manuscript received for publication April 7, 1983.) 